\title{
DOSSIER
}

\section{Neuropatia diabética}

TÂNIA ALMEIDA*, SÓNIA CUNHA CRUZ**

\section{RESUMO}

Introdução: A neuropatia periférica é a complicação mais comum da diabetes, capaz de comprometer todos os fecidos do corpo e ser causa de significativa morbilidade e mortalidade. Apesar da evolucão actual da ciência médica, continua complicada e incompleta a compreensão dos efeitos produzidos pelos processos patológicos da neuropatia diabética nas actividades fisiológicas normais e os tratamentos disponiveis são apenas modestamente eficientes no alivio dos sintomas.

Objectivo: Realizar uma revisão baseada na evidência, acerca dos actuais conhecimentos sobre patogénese, diagnóstico e tratamento da neuropatia diabética, para servir de base à elaboração de guidelines partuguesas.

Metodologia: Foi efectuada uma pesquisa bibliográfica na base de dados MEDLINE/Pubmed, de artigos de revisão publicados nos últimos 10 anos, através da utilização das palavras-chave "diabetic neuropathy» e "neuropatic pain». Foram incluídos no estudo 16 artigos.

Conclusões: 0 quadro clínico da neuropatia pode variar desde formas assinfomáticas até à presença de muitas manifestações pouco específicas, somáticas e/ou autonómicas, apresentando-se de duas formas principais: polineuropatia sensitivo-motora simétrica e neuropatia autonómica (cardiovascular, respiratória, digestiva e genitourinária). Menos frequentemente a lesão neuropática é mais localizada, apresentando-se nas formas de: mononeuropatia focal (tibiais, medianos, pares cranianos III, IV, VI e VII); neuropatia multifocal radicular (intercostal, toracoabdominal e lombar); neurqpotia multifocal multiplexos e plexopatia ou amiotrofia. A neuropatia diabética é uma complicação frequente da diabetes, multifacefada, que necessita de um leque de drogas muito bem administrado para o controle moderado dos sintomas. As medidas preventivas principais passam pelo bom controlo glicémico e pelo reconhecimento precoce das situações de risco.

Palavras-chave: Neuropatia Diabética; Neuropatia Autonómica; Neuropatia Somática; Dor Neuropática.

\section{INTRODUÇ̃̃O}

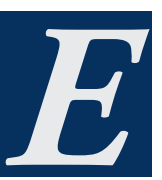

mbora a diabetes seja uma doença tão antiga como a humanidade e já tenha sido reconhecida há milhares de anos, como nos prova o papiro Ebers, documento egípcio escrito por volta de $1.500 \mathrm{AC}$, apenas há 2 séculos surgiram os primeiros trabalhos sobre as suas complicações neurológicas. Rollo (1798), Griesinger (1859) e Marchal de Calvi (1864) foram os pioneiros que relataram o aparecimento de lumbago $e$ ciática e o comprometimento do nervo ciático. Ogle (1866), apontou comprometimento dos nervos cranianos. Bouchard (1881), assinalou a abolição do reflexo patelar. Althaus, Pavy e Leval-Piquechet (1885), descreveram dores lan- cinantes e ataxia associadas à hiporreflexia, sendo este último autor o primeiro a referir os nervos periféricos como a sede da lesão. Charcot (1890), também admitiu a sua origem periférica ao estudar os problemas motores e deformações em pés de diabéticos. Williamson (1905), enfatizou a abolição da sensibilidade vibratória nos pés resultante do comprometimento dos nervos periféricos, reconhecendo-a como achado precoce numa doença - que denominou neurite periférica múltipla - e realçou a presença quase constante da perda do reflexo de Aquiles.

Com o passar do tempo, a literatura médica enriqueceu-se, merecendo destaque especial, nos últimos 30 anos, os estudos sobre o comprometimento do sistema nervoso autónomo na diabetes e a discussão sobre a sua etiopatogenia. $^{1}$

Apesar da actual evolução da ciência médica, a compreensão dos efeitos produzidos pelos processos patológicos da neuropatia diabética nas actividades fisiológicas normais continua complicada e incompleta. Os tratamentos disponíveis são apenas modesta a moderadamente eficientes no alivio dos sintomas, sendo a sua principal limitação os efeitos adversos e as interacções medicamentosas. Continuam a decorrer estudos experimentais e investigação terapêutica nesta área, em busca de novas fórmulas que intervenham no decurso natural da doença. ${ }^{2}$

Excluindo-se outras causas de neuropatia, sabe-se que a neuropatia periférica é a complicação mais comum da diabetes, capaz de comprometer todos os tecidos do corpo e ser causa de significativa morbilidade e mortalidade. Comprova-se que pode afectar cerca de 
$50 \%$ dos indivíduos com diabetes de longa duração (> 25 anos), sejam do tipo 1 ou 2. Esta prevalência pode aumentar até perto dos $100 \%$, quando se utilizam métodos diagnósticos de maior sensibilidade, como os electrofisiológicos. O ratio de incidência anual da doença é aproximadamente de $2 \% .^{2,3}$

Os distúrbios neurológicos podem ser detectados precocemente na diabetes tipo 2, muitas vezes desde o momento do diagnóstico, enquanto que na diabetes tipo 1 geralmente aparecem cinco ou mais anos após o diagnóstico. À luz dos conhecimentos actuais, sabe-se que o factor de risco primário é a hiperglicemia e o bom controlo metabólico da diabetes diminui a frequência e a intensidade da lesão neurológica. Estes aspectos foram demonstrados em grandes estudos prospectivos que envolveram indivíduos diabéticos tipo 1 e tipo 2.4.5

\section{OBJECTIVO}

As autoras propuseram-se realizar uma revisão baseada na evidência, acerca dos actuais conhecimentos sobre patogénese, diagnóstico e tratamento da neuropatia diabética, para servir de base à elaboração de guidelines nacionais.

\section{MetOdOLOGIA}

Foi efectuada uma pesquisa bibliográfica na base de dados Medline/Pubmed, de artigos de revisão e metanálises publicados nos últimos dez anos, com especial destaque nos últimos cinco anos, através da utilização das palavras-chave "diabetic neuropathy" e "neuropathic pain". Foram seleccionados os artigos cujo título ou resumo fossem de encontro aos objectivos do trabalho, tendo-se obtido posteriormente o texto integral. Foram incluídos no estudo 16 artigos.

\section{Definicões e Classificaç̃̃o}

As neuropatias caracterizam-se pela perda progressiva de fibras nervosas do sistema nervoso autónomo. A definição simples, internacionalmente aceite, de neuropatia diabética é «a presença de sinais e/ou sintomas de disfunção nervosa periférica, em doentes com diabetes, após exclusão de outras causas». ${ }^{6}$ Um subtipo de polineuropatia periférica, a neuropatia diabética autonómica, pode envolver todo o sistema nervoso autónomo. Esta entidade é difícil de reconhecer e pode estar presente de forma clínica ou subclínica, mesmo no primeiro ano de diagnóstico na diabetes tipo 2 ou após dois anos de evolução na diabetes tipo $1 .{ }^{7}$ Pode manifestar-se por disfunção em apenas um ou em vários órgãos e sistemas, sendo os mais frequentemente afectados o cardiovascular, o gastrointestinal, o genitourinário e o ocular.

Ao longo dos anos têm sido propostas várias classificações para a grande variedade de síndromes afectando o sistema nervoso periférico na diabetes. Algumas baseiam-se nos factores etiopatogénicos, mas como ainda não são conhecidos todos os mecanismos envolvidos, as classificações clínicas são as mais utilizadas. ${ }^{8} \mathrm{O}$ Quadro I apresenta a classificação adoptada nesta revisão.

\begin{tabular}{|c|}
\hline QUADRO I \\
\hline CLASSIFICAC̣ÃO DA NEUROPATIA DIABÉTICA \\
\hline Polineuropatias simétricas generalizadas \\
\hline Sensitivo-motora (crónica) \\
\hline Sensitiva aguda \\
\hline Autonómica \\
\hline Neuropatias focais e multifocais \\
\hline Craniana \\
\hline Radiculoneuropatia toraco-lombar \\
\hline Focal dos membros \\
\hline Motora proximal (amiotrofia) \\
\hline
\end{tabular}




\section{ETIOPATOGENIA}

Vários factores estão implicados na génese da neuropatia diabética, sendo o principal a hiperglicemia. Ficou demonstrado que o bom controlo metabólico, através da terapêutica intensiva com insulina, melhora a neuropatia em diabéticos tipo $1 .{ }^{4}$ No entanto, esse beneficio, apesar de também ser real, é menor na diabetes tipo $2 .{ }^{5}$ Outros mecanismos estão também implicados na patogénese desta situação, nomeadamente a activação da via poliol, levando à acumulação de sorbitol, com consequente alteração do NAD (nicotinamida adenina dinucleotídeo) para NADH (forma reduzida do NAD), levando a lesão do nervo. ${ }^{7}$

A diabetes é um estado essencialmente hipermetabólico, como tal promove a entrada celular de elevadas concentrações de glicose. Esta elevada concentração de açúcar leva à formação de produtos de glicação avançada, que por sua vez aumentam o stress oxidativo celular. ${ }^{2}$ Existem alguns estudos em curso sobre o papel dos factores de crescimento neste processo, nomeadamente NGF (nerve growth factor), IGF-1 (insulin-like growth factor), VEGF (endothelium-specific growth factor), entre outros. Foi também proposta, por alguns autores, a presença de uma imunoglobulina auto-imune nos diabéticos tipo 2 , que estimula a apoptose das células nervosas pela activação do complemento. ${ }^{7}$ Todos estes factores estão ainda em estudo mas sabe-se que, em conjunto com a hiperglicemia, desempenham um papel importante na lesão vascular e nervosa, levando ao aparecimento de neuropatia.

\section{Caracterizaç̃o da Doença}

A lesão neurológica na neuropatia diabética envolve amplamente todo o sistema nervoso periférico, nos seus componentes sensório-motor e autonómico, com clínica característica e concordante com as hipóteses patogénicas de natureza metabólica e/ou microvascular. Nos estudos realizados em grupos de diabéticos utilizando metodologia clínica, verificou-se predominância da neuropatia sensitivo-motora. Este achado pode, no entanto, dever-se à própria metodologia empregue, já que os testes de função autonómica são de utilização mais dificil por envolverem métodos e equipamentos mais sofisticados. ${ }^{9}$

Desse modo, o quadro clínico da neuropatia pode variar desde formas assintomáticas até à presença de muitas manifestações pouco específicas, somáticas e/ou autonómicas, apresentando-se de duas formas principais: polineuropatia sensitivo-motora simétrica e neuropatia autonómica (cardiovascular, respiratória, digestiva, genitourinária). Menos frequentemente a lesão neuropática é mais localizada, apresentando-se nas formas de: mononeuropatia focal (tibiais, medianos, pares cranianos III, IV, VI e VII); neuropatia multifocal radicular (em geral intercostal, toraco-abdominal e lombar); neuropatia multifocal multiplexos (localização variada) e plexopatia ou amiotrofia..$^{9-11}$

\section{DIAGNÓSTICO}

O diagnóstico das formas mais frequentes de neuropatia diabética baseia-se na caracterização do quadro clínico, com os sinais e sintomas mais típicos, e na realização de testes neurológicos. As principais manifestações clínicas de comprometimento somático são a dormência ou sensação de queimadura nos membros inferiores, formigueiro, pontadas, choques, agulhadas nas pernas e pés (parestesias), desconforto ou dor ao toque (por exemplo, lençóis e cobertores - alodinia) e queixas de diminuição ou perda da sensibilidade táctil, térmica ou dolorosa. Apesar dos sintomas serem mais frequentes nos membros 
inferiores e pés os membros superiores também podem ser afectados. É importante referir que a ausência dos sinais e sintomas descritos não exclui a presença de neuropatia, pois alguns doentes evoluem directamente para a perda total de sensibilidade. ${ }^{11}$

Os testes neurológicos básicos envolvem a avaliação de sensibilidade, pesquisa de reflexos tendinosos, medição da pressão arterial (deitado e em pé) e da frequência cardíaca:

1. Avaliação da sensibilidade dolorosa (palito ou agulha), táctil (algodão ou monofilamento), térmica (quente/ /frio) e vibratória (diapasão de 128 $\mathrm{Hz}$;

2. Pesquisa de reflexos tendinosos (aquiliano, patelar e tricipital);

3. Medida da pressão arterial sistémica em posição deitada e ortostática (hipotensão postural: queda da pressão arterial sistólica $>20 \mathrm{mmHg}$ um minuto após assumir posição ortostática);

4. Frequência cardíaca de repouso sugestiva de disautonomia cardiovascular quando acima de 100 ppm.

Outros testes neurológicos mais complexos e de dificil realização por rotina $^{12,13}$ confirmam a lesão neurológica:

1. Testes sensoriais quantitativos: avaliação da neurocondução, especialmente em membros inferiores.

2. Testes da regulação cardiovascular: medidas do intervalo RR, manobra de Valsalva, teste postural passivo, arritmia sinusal respiratória e esforço isométrico.

3. Medidas directas da integridade simpática cardíaca: cintigrafia com metaiodobenzilguanidina e tomografia por emissão de positrões (PET) com 11-c-hidroxiefedrina.

\section{TRATAMENTO}

Actualmente ainda não existe um tratamento eficaz a longo prazo para tra- tar as várias formas de neuropatia. Existem alguns estudos em curso $^{11} \mathrm{com}$ o objectivo de testar fármacos que actuem nos mecanismos etiopatogénicos da doença. Infelizmente, estes ainda não estão disponíveis e os seus resultados têm sido pouco ou nada significativos.

O que a seguir se descreve são as actuais opções disponiveis para os vários tipos de neuropatia.

\section{Controlo metabólico}

O bom controlo metabólico é sem dúvida o principal factor preventivo da neuropatia, ${ }^{2}$ quer prevenindo o aparecimento da lesão, quer a sua intensidade e extensão. Alguns estudos ${ }^{4}$ também sugerem que o bom controlo metabólico pode melhorar a neuropatia já estabelecida.

\section{Tratamento dos sinais e sintomas} da neuropatia sensitivo-motora

A maioria dos profissionais de saúde reconhece como neuropatia diabética a dor torturante e refractária que apenas ocorre numa minoria (11-32\%) de diabéticos com neuropatia sensitivo-moto$\mathrm{ra}^{2}$ e não está correlacionada com a diminuição da condução da velocidade ou da função do nervo. Contudo, a destruição nervosa típica desenvolve-se de forma insidiosa e geralmente assintomática, levando a perda ou a alterações da sensibilidade que só podem ser detectadas e quantificadas por testes clínicos específicos. A neuropatia sensitivo-motora é o principal factor de risco para o desenvolvimento de úlceras no pé diabético, que por sua vez são responsáveis por cerca de $85 \%$ das amputações ocorridas em pacientes diabéti$\cos ,{ }^{2,9,11}$ pelo que o investimento na prevenção e no bom controlo metabólico é fundamental. A Figura 1 apresenta um algoritmo para o tratamento deste tipo de neuropatia.

As principais opções terapêuticas para as parestesias e dor ${ }^{8,14}$ da neuro- 


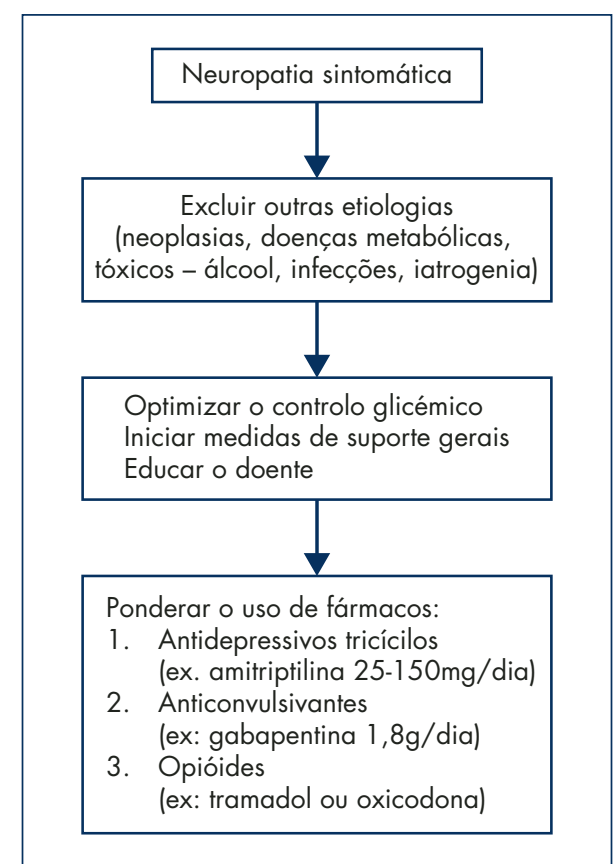

Figura 1. Abordagem da neuropatia diabética periférica

patia diabética são:

1. Medicamentos antidepressivos tricíclicos: amitriptilina (25-150 mg), imipramina (25-150 mg) e nortriptilina (25-150 mg) por via oral/dia.

2. Medicamentos anticonvulsivantes: carbamazepina (200-800 mg), gabapentina (900-1800mg), pregabalina (50-200 mg) e lamotrigina (50-400 $\mathrm{mg}$ ) por via oral/dia.

3. Outros fármacos: paroxetina (40 mg), citalopram (40 mg), tramadol (50-400 $\mathrm{mg}$ ) e oxicodona (10-60 mg) por via oral/dia.

4. Analgésicos tópicos: capsaicina $(0,075 \%)$ creme; lidocaína gel $(5 \%)$.

5. Acupunctura: em alternativa ou complemento (ainda em investigação).

Tratamento dos sinais e sintomas da neuropatia autonómica

Por existirem vários quadros clínicos de neuropatia autonómica, o tratamento depende da localização e dos sistemas afectados. O Guadro II apresenta um resumo do tratamento de acordo com a sintomatologia existente.

\section{Disautonomia caRDIOVASCULAR (OU NEUROPATIA AUTONÓMICA CARDIOVASCULAR)} A prevenção da disautonomia cardiovascular é essencial, porque há poucos tratamentos eficientes para a doença já estabelecida. No entanto, no seu estádio inicial, pode ser revertida pelo controlo eficiente da glicemia, existindo evidência de que o seu risco pode ser reduzido em $68 \%$ através do controle da hiperglicemia, da dislipidemia e da hipertensão. ${ }^{2,15} \mathrm{O}$ desequilíbrio entre a actividade simpática e parassimpática pode ser estabilizado ou melhorado com a utilização de inibidores da enzima de conversão da angiotensina (IECA's) e beta-bloqueantes, como o atenolol, o metoprolol e o propanolol. ${ }^{2,7,9}$

Os sintomas da hipotensão postural podem ser reduzidos com medidas não farmacológicas, como sejam: evitar mudanças posturais bruscas, uso de meias ou calças compressivas, elevação da cabeceira do leito $(30 \mathrm{~cm})^{7}$

\section{DISAUTONOMIA GASTROINTESTINAL (OU NEUROPATIA AUTONÓMICA GASTROINTESTINAL)}

O tratamento primário da disautonomia gastrointestinal passa pelo bom controlo glicémico, que no entanto se torna muito frustrante, já que a própria patologia modifica o tempo da absorção dos nutrientes e da terapêutica anti-diabética. A hiperglicemia tem sido demonstrada como causa directa da redução e atraso das contracções gástricas e do esvaziamento gastrointestinal. Está também relatada uma sensação de saciedade e plenitude gástrica com níveis elevados de glicemia. No entanto, alguns estudos têm demonstrado que a redução a curto prazo, apenas da hiperglicemia pré-prandial, não conduz à resolução dos sintomas, que são mais prevalentes nos doentes com mau controlo glicémico.9.16

O tratamento não farmacológico in- 


\section{DossIER}

\section{QUADRO II}

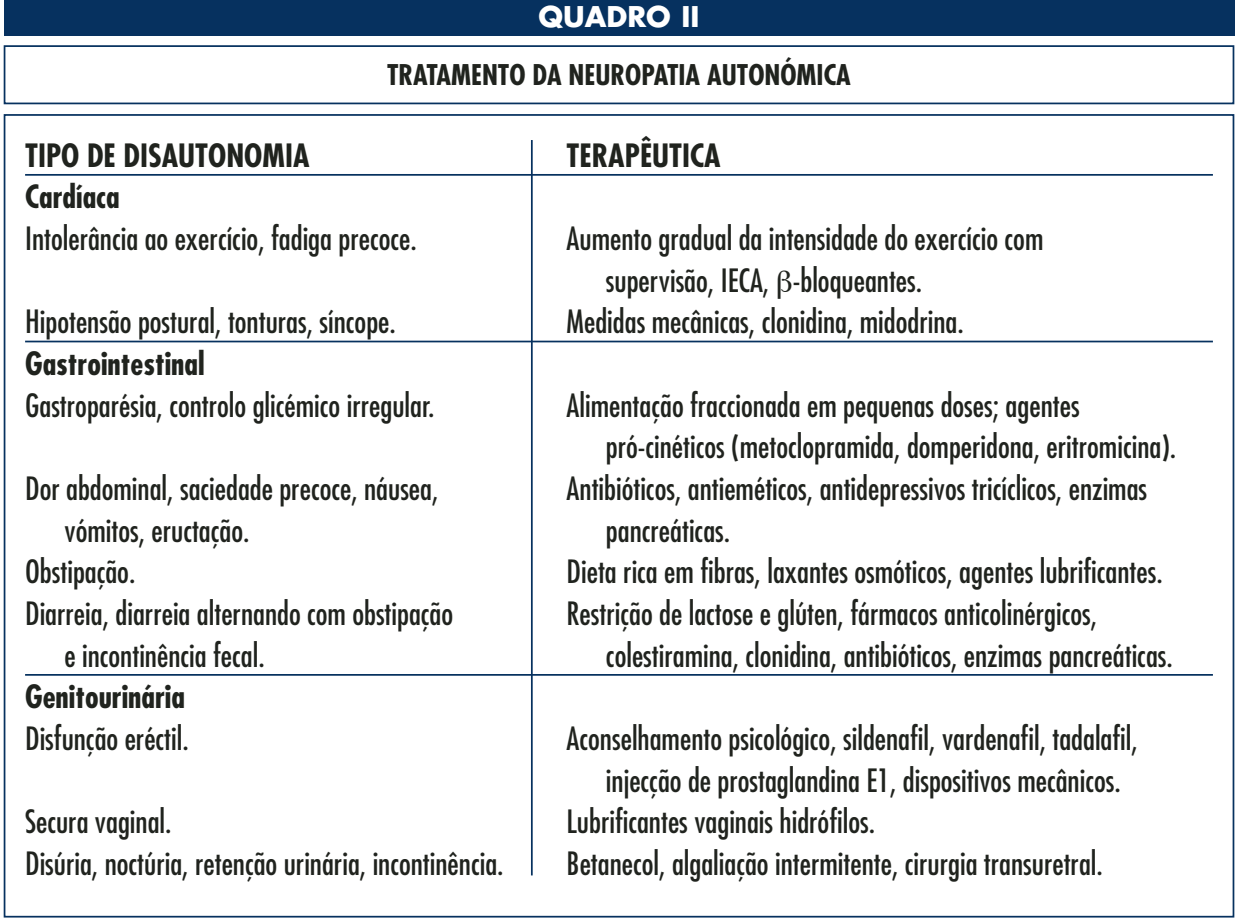

cide directamente na modificação alimentar, sendo recomendável que doentes sintomáticos reduzam o conteúdo de gorduras e aumentem a ingestão de fibras. ${ }^{16}$

Para alívio das crises de enfartamento pós-prandial, obstipação e diarreia podem utilizar-se as seguintes opções terapêuticas:

- Gastroparésia: metoclopramida, cisapride ou domperidona;

- Obstipação: aumento da ingestão de fibras;

- Diarreia: antibióticos de largo espectro e loperamida.

\section{DisAuTONOMIA GENITOURINÁRIA (OU} NEUROPATIA AUTONÓMICA GENITOURINÁRIA) A bexiga neurogénica geralmente não responde bem ao tratamento farmacológico. A retenção urinária daí resultante representa um elevado risco de infecções urinárias de repetição. A utilização de técnicas de esvaziamento torna-se então necessária, recorrendo em algumas situações à cateterização vesical. ${ }^{2,3}$
O tratamento da disfunção sexual começa sempre pelo bom controlo glicémico. Vários estudos demonstraram existir uma relação directa entre a disfunção eréctil e a elevação da hemoglobina Alc. ${ }^{2,10,17}$ A prevalência da disfunção sexual feminina é mais elevada na mulher diabética que na população em geral. Esta patologia não tem sido adequadamente estudada ${ }^{16} \mathrm{e}$ os tratamentos são virtualmente desconhecidos, embora a secura vaginal e a dispareunia possam responder bem ao uso de lubrificantes hidrófilos. ${ }^{2}$

Nas situações de bexiga neurogénica deve ser aconselhado o treino de esvaziamento vesical programado, completo com manobras de compressão abdominal e auto-algaliação. Em alguns casos há necessidade de instituir antibioterapia para tratar ou prevenir as infecções urinárias. ${ }^{2,17}$ Actualmente, a primeira escolha para o tratamento da disfunção eréctil são os medicamentos do grupo dos inibidores da fosfodiasterase (sildenafil, vardenafil e tadalafil). 
Em situações mais raras, também podem ser utilizadas drogas de uso intracavernoso ou intra-uretral (papaverina, fentolamina e prostaglandinas), prótese peniana e dispositivos a vácuo. ${ }^{2,17}$

\section{RECOMENDACÕ̃ES PARA TRATAMENTO E RASTREIO}

A principal recomendação da American Diabetes Association é o controlo glicémico apertado ${ }^{11}$ para todos os doentes diabéticos, mantendo também os níveis de $\mathrm{HbAlc}$, tensão arterial e lípidos dentro dos valores recomendados, recorrendo para tal à modificação dos estilos de vida e à instituição de farmacoterapia.

$\mathrm{O}$ rastreio da neuropatia periférica sensitivo-motora está preconizado em todos os doentes com diabetes tipo 2 , a partir do diagnóstico e 5 anos após o diagnóstico nos diabéticos tipo 1. A frequência deve ser anual ou semestral, através da história clínica e do exame físico, averiguando a sensibilidade nos pés e procedendo à pesquisa dos reflexos osteo-tendinosos do membro inferior. Podem ser utilizados os testes de sensibilidade térmica, percepção da pressão (utilizando o monofilamento de Semmes-Weinstein), percepção vibratória (utilizando o diapasão de $128 \mathrm{~Hz}$ ) ou sensibilidade táctil. $\mathrm{O}$ pé deve ser inspeccionado para detecção de úlceras, calosidades ou deformações. Todos os doentes com neuropatia periférica, seja ou não sintomática, devem ser educados sobre a sua situação clínica e referenciados para o podologista.

O rastreio da neuropatia autonómica deve ser aplicado ao mesmo grupo de doentes anteriormente descrito e com a mesma frequência. ${ }^{11}$ Deve fazer parte do rastreio a história clínica e o exame físico, com particular atenção para os sinais de disfunção autonómica já descritos neste artigo. Os testes mais indicados no rastreio são a pesquisa de variabilidade da frequência cardíaca, incluindo a resposta às manobras de Valsalva e ortostatismo. ${ }^{11,15} \mathrm{Se}$ estes testes forem positivos, será apropriado realizar outros mais específicos e iniciar medidas terapêuticas.

\section{O Papel do Médico de Famíla}

O envolvimento neuropático dos nossos doentes diabéticos é geralmente uma complicação precoce e de alta prevalência (10 a $100 \%$ ), sendo a maior da triopatia diabética - retinopatia, nefropatia e neuropatia. ${ }^{13,14}$ Constitui um importante problema de saúde, com elevada morbilidade e mortalidade, piorando significativamente a qualidade de vida, por incapacidade, e causando diminuição da sobrevida. A sua etiopatogenia continua desconhecida e complicada, mesmo para o conhecimento médico actual. Os tratamentos disponiveis para esta entidade clínica proporcionam apenas alívio moderado dos sintomas, sendo limitados pelos efeitos adversos e pelas interacções medicamentosas. ${ }^{2}$ Todos os estudos actuais ${ }^{2-5}$ demonstram que o bom controlo metabólico da diabetes evita ou atrasa o aparecimento da neuropatia diabética e diminui a frequência e a intensidade da lesão neurológica. Como tal, o médico de família tem uma importância crucial no diagnóstico precoce dos doentes em risco, na instituição das medidas preventivas possiveis e na boa gestão do controlo do doente.

Desde o momento do diagnóstico, cabe-nos a nós, como médicos de família, a responsabilidade de educar os nossos doentes diabéticos sobre a doença e as suas complicações agudas e crónicas. ${ }^{6.14}$

É preciso enfatizar que a falha no diagnóstico da neuropatia diabética pode trazer sérias consequências, incluindo incapacidade, amputação e 
morte. Uma das responsabilidades do médico de família é tratar o impacto psicológico da disfunção sexual, tanto no homem como na mulher, e não apenas os factores físicos. Por vezes é necessário o envolvimento do neurologista (preferencialmente com especialização em neuropatia periférica) no tratamento do doente com neuropatia diabética, mas este não deve ser sobrevalorizado. $\mathrm{O}$ médico de família, atento a esta situação, pode e deve fazer a sua correcta abordagem e tratamento.

\section{CONCLUSÕES}

A neuropatia diabética é uma complicação frequente da diabetes, multifacetada, causa de significativa morbilidade e mortalidade, que necessita de um leque de drogas muito bem administrado para ter as suas manifestações modestamente controlados. As medidas preventivas principais passam pelo bom controlo glicémico e pelo reconhecimento precoce das situações de risco. O médico de família pode e deve ser um elemento chave para o sucesso da prevenção desta entidade clínica.

\section{REFERÊNCIAS BIBLIOGRÁFICAS}

1. Pompeu F. Neuropatia diabética. In: Arduino F, editor. Diabetes Mellitus. Rio de Janeiro:Editora Guanabara-Koogan; 1980. p. 348.

2. Duby JJ, Campbell RK, Setter SM, White JR, Rasmussen KA. Diabetic neuropathy: an intensive review Am J HealthSyst Pharm 2004 Jan 15; 61 (2): 160-73.

3. Simmons Z, Feldman EL. Update on diabetic neuropathy. Curr Opin Neurol 2002 Oct; 15(5): 595-603.

4. Writing Team for the Diabetes Control and Complications Trial / Epidemiology of Diabetes Interventions and Complication Research Group. Effect of intensive therapy on the microvascular complications of type 1 diabetes mellitus. JAMA 2002 May 15; 287 (19): 2563-9.

5. UK Prospective Diabetes Study Group (UKPDS). Intensive blood-glucose control with sulphonylureas or insulin compared with conventional treatment and risk of complications in patients with type 2 (UKPDS 33). Lancet 1998 Sep 12; 352 (9131): 837-53.

6. Boulton AJ, Gries FA, Jervell JA. Guidelines for the diagnosis and outpatient management of diabetic peripheral neuropathy. Diabet Med 1998 Jun; 15 (6): 508-14.

7. Vinik AI, Maser RE, Mitchell BD, Freeman R. Diabetic autonomic neuropathy. Diabetes Care 2003 May; 26 (5): 1553-79.

8. Boulton AJ, Malik RA, Arezzo JC, Sosenko JM. Diabetic somatic neuropathies. Diabetes Care 2004 Jun; 27 (6): 1458-86.

9. Martin CL, Albers J, Herman WH, Cleary P, Waberski B, Greene DA, et al. Neuropathy among the diabetes control and complications trial cohort 8 years after completion. Diabetes Care 2006 Feb; 29 (2): 340-4.

10. Pirart J. Diabetes mellitus and its degenerative complications: a prospective study of 4,400 patients observed between 1947 and 1973. Diabetes Care 1978 May; 1 (3): 168-88.

11. Boulton AJ, Vinik AI, Arezzo JC, Bril V, Feldman EL, Freeman R, et al. Diabetic neuropathies: a statement by the American Diabetes Association. Diabetes Care 2005 Apr; 28 (4): 956-62.

12. Dyck, PJ, Kratz, KM, Lehman, KA, Karnes JL, Melton LJ 3rd, O'Brien PC, et al. The Rochester Diabetic Neuropathy Study: design, criteria for types of neuropathy, selection bias, and reproductibility of neuropathic tests. Neurology 1991 Jun; 41 (6): 799-807.

13. Suarez GA, Clark VM, Norell JE, Kottke TE, Callahan MJ, O'Brien PC, et al. Sudden cardiac death in diabetes mellitus: risk factors in the Rochester diabetic neuropathy study. J Neurol Neurosurg Psychiatry 2005 Feb; 76 (2): 240-5.

14. Waldman SD. Diabetic neuropathy: diagnosis and treatment for the pain management specialist. Curr Rev Pain 2000; 4 (5): 383-7.

15. Vinik AI, Ziegler D. Diabetic cardiovascular autonomic nuropathy. Circulation 2007 Jan 23; 115 (3): 387-97.

16. Jones KL, Russo A, Berry MK, Stevens JE, Wishart JM, Horowitz M. A longitudinal study of gastric emptying and upper gastrointestinal symptoms in patients with diabetes mellitus. Am J Med 2002 Oct 15; 113 (6): 449-55 .

17. Brown JS, Wessells H, Chancellor MB, Howards SS, Stamm WE, Stapleton AE, et al. Urologic complications of diabetes. Diabetes Care 2005 Jan; 28 (1): 177-85. 


\section{DossIER}

\section{DIABETIC NEUROPATHY}

\section{ABSTRACT}

Introduction: Peripheral neuropathy is the most common complication of diabetes, affecting all tissues and causing significant morbility and mortality. In spite of current medical progress, our understanding of the effects of diabetic neuropathy on normal physiological activities is still incomplete and available treatments are only modestly efficient for symptom relief.

Objective: To review the current literature on diabetic neuropathy, its pathogenesis, diagnosis and treatment as a basis for the creation of Portuguese guidelines for the management of this condition.

Methodology: A MEDLINE/Pubmed search was conducted of review published in the last 10 years using the following key-words: "diabetic neuropathy" and "neuropathic pain". Sixteen articles were found using this strategy.

Conclusions: The clinical picture of diabetic neuropathy can vary from asymptomatic forms to nonspecific, somatic or autonomic forms. These may present in two main forms: a symmetrical sensorimotor polyneuropathy and an autonomic polyneuropathy (cardiovascular, respiratory, digestive and urogenital). Less commonly, the neuropathic lesion is more localized, presenting in the form of a focal mononeuropathy (tibial, median, or in cranial nerves III, IV, VI and VII); radicular multifocal neuropathy (intercostal, thoracoabdominal and lumbar), multifocal neuropathy, plexopathy or amyotrophy. Diabetic neuropathy is a frequent complication of diabetes. It is multifacefed, and requires a wide range of drugs in order to achieve moderate symptom control. The main preventive measures are good glycemic control and early recognition.

Key-words: Diabetic Neuropathy; Autonomic Neuropathy; Somatic Neuropathy; Neuropathic Pain.

\section{Endereço para correspondência:}

Tânia Almeida

Centro de Saúde Vialonga

Urbanização Olival da Fonte, lote 19

2625-623 Vialonga

E-mail: tpralmeida@gmail.com

Sónia Cunha Cruz

CS São João da Talha

Rua Maria Conceição Costa

2685 S João da Talha

E-mail: sonicruz@yahoo.com 